\title{
Increasing Spatial Resolution and Extending Spectral Range of Synchrotron Infrared Microscopy
}

\author{
Gregory D. Smith* and G. L. Carr* \\ *National Synchrotron Light Source, Brookhaven National Laboratory, P. O. Box 5000, Upton, NY \\ 11973-5000
}

Conventional IR microscopes have found widespread use in identifying the chemical content of microsamples and in mapping the distribution of materials in heterogeneous samples based on characteristic infrared absorptions. These instruments, which typically employ Schwarzschild reflecting optics and thermal sources, are capable of recording IR spectra in the region of 2.5 to 15 $\mu \mathrm{m}$ (4000 to $670 \mathrm{~cm}^{-1}$ ) with a spatial resolution of $\sim 20 \mu \mathrm{m}$ while maintaining reasonably good signal-to-noise ratios. The spatial resolution of IR microscopes is significantly improved when they are coupled to a synchrotron radiation infrared (SR-IR) source; the high brightness and low beam divergence of SR-IR allow diffraction limited performance $(\sim \lambda) .[1]$

This paper describes recent improvements to the U4IR beamline and associated instrumentation at the National Synchrotron Light Source that have extended the long wavelength spectral range of that facility to $\sim 200 \mu \mathrm{m}\left(50 \mathrm{~cm}^{-1}\right)$ while maintaining its performance in the mid-IR. A mapping stage capable of sub-micron positioning allows detailed measurements of the diffraction pattern (Figure 1) that can be used to test deconvolution methods for increasing the spatial resolution. As an example, an absorption profile at $1600 \mathrm{~cm}^{-1}$ for a line scan across a sharp-edged target is shown in Figure 2 . Also shown is the calculated profile assuming the diffraction pattern of Figure 1. The agreement between the calculated values and the experimental result demonstrates that diffraction-limited performance is achieved by SR-IR and motivates the deconvolution method for enhancing spatial resolution. The high brightness of SR-IR also permits the use of small confocal apertures for microscopy, which should yield additional improvements to the spatial resolution and contrast. In addition, confocal aperturing imparts the vertical spatial discrimination necessary for depth profiling of material inclusions (see Figure 3). The use of high refractive index materials ( $\mathrm{ZnSe}, \mathrm{Ge}$, Diamond, \& Si) for attenuated total internal reflection (ATR) and transmission sampling accessories can similarly improve the spatial resolution of SR-IR microscopy by up to a factor of four [2]. The study of these optical and deconvolution techniques in combination is currently underway and further advances will be presented, along with examples of SR-IR microscopy applications to the identification and study of cosmic dust particles, artists' materials, and other inorganic and organometallic samples.

The NSLS is operated as a User Facility for the U.S. Department of Energy under contract DEAC02-98CH10886. Requests for access to the facility are welcome. Information regarding the procedures for research proposals are available at http://WwW.nsls.bnl.gov or by contacting the program director by email: carr@bnl.gov. 


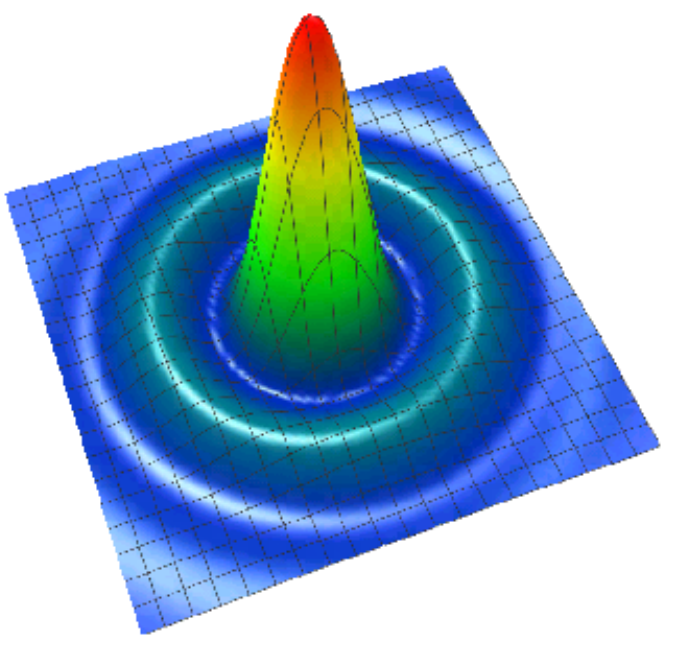

Figure 1. Calculated diffraction pattern for a model Schwarzschild microscope objective showing an enhanced $1^{\text {st }}$ order diffraction "ring."

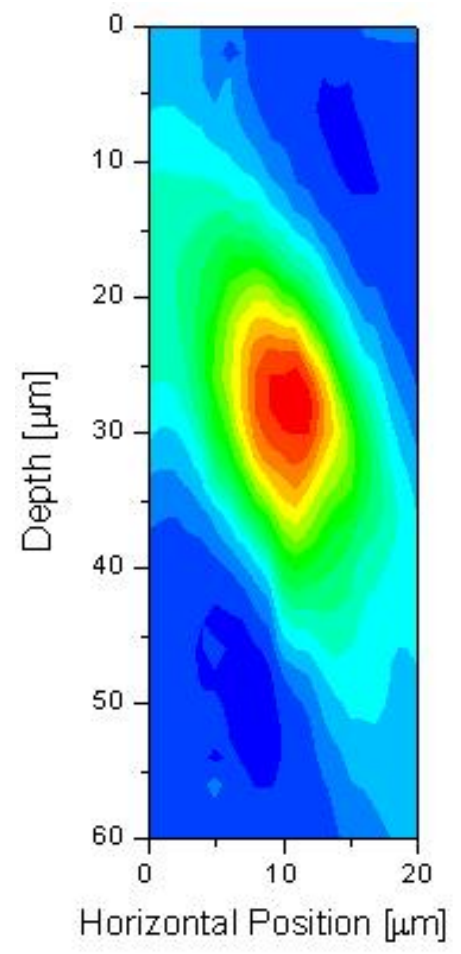

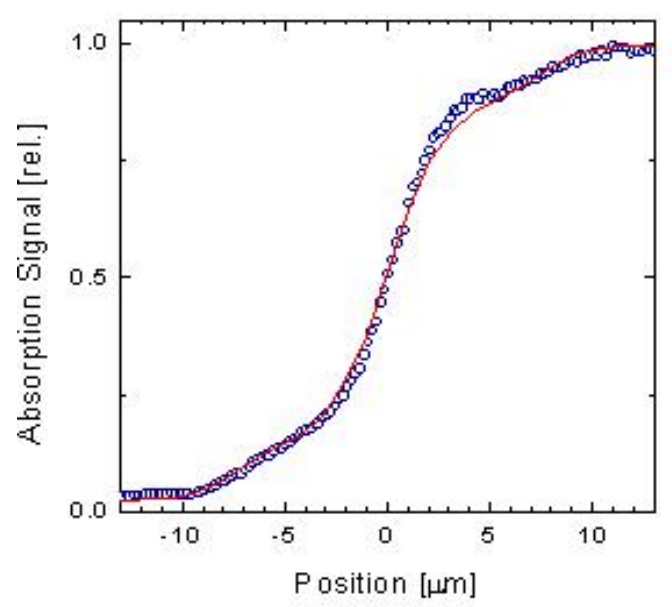

Figure 2. Calculated (red line) and measured (blue circles) edge profile for the amide I vibration $(\lambda=6 \mu \mathrm{m})$ under diffraction-limited conditions.

Figure 3. Vertical depth profile of a $6 \mu \mathrm{m}$ diameter Te particle imbedded in a $\mathrm{Cd}(\mathrm{Zn}) \mathrm{Te}$ wafer, imaged using confocal SR-IR microscopy. The false color value is determined by the absorption at $\lambda=3 \mu \mathrm{m}$.

\section{References}

[1] G. L. Carr, Rev. Sci. Instr. 72 (2001) 1.

[2] A. J. Sommer et al., Appl. Spectrosc. 55 (2001) 252. 